\title{
Transient average constraints in economic model predictive control $^{\star}$
}

\author{
Matthias A. Müller a David Angeli b,c Frank Allgöwer ${ }^{\text {a }}$ \\ ${ }^{\mathrm{a}}$ Institute for Systems Theory and Automatic Control, University of Stuttgart, 70550 Stuttgart, Germany. \\ ${ }^{\mathrm{b}}$ Department of Electrical and Electronic Engineering, Imperial College, London, UK. \\ ${ }^{\mathrm{c}}$ Dip. di Ingegneria dell'Informazione, University of Firenze, Italy.
}

\begin{abstract}
In this paper, an economic model predictive control algorithm is proposed which ensures satisfaction of transient average constraints, i.e., constraints on input and state variables averaged over some finite time period. We believe that this stricter form of average constraints (compared to previously proposed asymptotic average constraints) is of independent interest in various applications such as the operation of a chemical reactor, where e.g. the amount of inflow or the heat flux during some fixed period of time must not exceed a certain value. Besides guaranteeing fulfillment of transient average constraints for the closed-loop system, we show that closed-loop average performance bounds and convergence results established in the setting of asymptotic average constraints also hold in case of transient average constraints. Furthermore, we illustrate our results with a chemical reactor example.
\end{abstract}

\section{Introduction}

Economic model predictive control (MPC) is a recently introduced variant of MPC, where the stage cost function used within the repeatedly solved optimization problem does not necessarily have to be positive definite with respect to any setpoint (or trajectory), as is the case in standard tracking MPC. A motivation for studying this more general MPC framework comes from applications where, e.g., the economics of a process should be optimized. In such cases, the (economic) cost function is typically not related to, and hence also not positive definite with respect to, any specific steady-state. In [27], different economic MPC settings have been studied, using different assumptions and/or additional (terminal) constraints. Furthermore, various applications of economic MPC were reported recently, see, e.g., [8-10].

One of the major strengths of MPC is the ability to

^ A preliminary version of parts of this paper was pre-
sented at the 52nd IEEE Conference on Decision and Con-
trol (CDC), Florence, Italy, 2013 (see [1]). The work of the
first and third author was supported by the German Re-
search Foundation (DFG) within the Priority Programme
1305 "Control Theory of Digitally Networked Dynamical
Systems" and within the Cluster of Excellence in Simulation
Technology (EXC 310/1) at the University of Stuttgart.
Email addresses:
matthias.mueller@ist.uni-stuttgart.de (Matthias A.
Müller), d.angeli@imperial.ac.uk (David Angeli),
frank.allgower@ist.uni-stuttgart.de (Frank Allgöwer). explicitly incorporate constraints into the controller design. In standard tracking MPC, pointwise in time state and input constraints are typically of interest. These are imposed on predicted (virtual) input/state signals, and hence are, by definition of the receding horizon control law, also satisfied for the actual closed-loop signals. On the other hand, besides pointwise in time constraints, also constraints on asymptotic average values of states and inputs become of interest in economic MPC and were recently studied in $[2,7]$. Namely, in standard tracking MPC with guarantee of convergence to an equilibrium setpoint, any asymptotic average of state and input variables is determined by their value at this equilibrium. Therefore, asymptotic average constraints are not of further interest online (i.e., within the design of the receding horizon controller), but have to be taken into account offline when determining the setpoint to be stabilized. On the other hand, one of the key features of economic MPC is that the closed-loop system may not converge to some steady-state, but might exhibit a periodic or even complex behavior, which is due to the use of a general (non positive definite) cost function. Hence it is natural to also consider constraints on average values of input and state variables, which now have to be taken into account online, i.e., within the MPC algorithm.

In this paper, we propose an economic MPC algorithm which ensures satisfaction of transient average constraints. This means that constraints are imposed on input and state variables averaged over some finite time period $T$, in contrast to asymptotic average con- 
straints $[2,7]$ which only have to hold asymptotically. By the same reasoning as above, such constraints are in particular meaningful (and have to be taken into account online) within the framework of economic MPC; however, the same is now also true for the initial phase (i.e., before converging) in tracking MPC. We believe that the concept of transient average constraints is of interest in various applications such as the operation of a chemical reactor, where e.g. the amount of inflow or the heat flux through the reactor wall during a given time period must not exceed a certain value (such an example is considered in Section 4). Our main contribution (see Theorem 1 in Section 3) is to show that a suitably defined economic MPC algorithm will ensure satisfaction of transient average constraints for the closed-loop system. This result is based on the assumption that the transient average constraints are satisfied inside the terminal region; if this assumption does not hold, we show that the closed-loop system satisfies a relaxed form of the original transient average constraints (see Section 3.1). Furthermore, we show that closed-loop average performance bounds and convergence results under a dissipativity condition, which were established in $[7,11]$ in a setting with asymptotic average constraints, can be transferred to our framework with transient average constraints (see Theorem 2 in Section 3). We apply the concept of transient average constraints to a chemical reactor in Section 4, and conclude the paper in Section 5 . We close this section by noting that a preliminary version of parts of the results in this paper has been presented in the conference paper [1]. The main novelties of the present paper compared to [1] are the consideration of relaxed transient average constraints in Section 3.1 as well as the chemical reactor example in Section 4.

\section{Preliminaries and setup}

\subsection{Notation}

Let $\mathbb{I}_{[a, b]}$ denote the set of integers in the interval $[a, b] \subseteq$ $\mathbb{R}$, and $\mathbb{I}_{\geq a}$ the set of integers greater or equal than $a$. For $a \in \mathbb{R},\lfloor a\rfloor$ is defined as the largest integer smaller or equal to $a$. The point to set distance of a point $x \in \mathbb{R}^{n}$ to a set $\mathbb{Y} \subseteq \mathbb{R}^{n}$ is defined as $|x|_{\mathbb{Y}}:=\inf _{y \in \mathbb{Y}}|x-y|$. Given two sets $\mathcal{A}, \mathcal{B} \subseteq \mathbb{R}^{n}$, the Minkowski set addition is defined as $\mathcal{A} \oplus \mathcal{B}:=\{a+b: a \in \mathcal{A}, b \in \mathcal{B}\}$. For a symmetric matrix $S \in \mathbb{R}^{n \times n}$, let $\lambda_{\min }(S)$ and $\lambda_{\max }(S)$ denote its minimum, respectively maximum, eigenvalue. As in [2], for any vector valued bounded signal $v: \mathbb{I}_{\geq 0} \rightarrow$ $\mathbb{R}^{n_{v}}$, the set of asymptotic averages is defined as $A v[v]:=$ $\left\{\bar{v} \in \mathbb{R}^{n_{v}}: \exists t_{n} \rightarrow+\infty\right.$ s.t. $\lim _{n \rightarrow \infty} \sum_{k=0}^{t_{n}-1}\left(1 / t_{n}\right) v(k)=$ $\bar{v}$. Note that $A v[v]$ is nonempty (as bounded sequences in $\mathbb{R}^{n_{v}}$ have limit points), but it need not be a singleton.

\subsection{Problem setup}

We consider discrete-time nonlinear systems of the form

$$
x(t+1)=f(x(t), u(t)), \quad x(0)=x_{0},
$$

with $t \in \mathbb{I}_{\geq 0}$, where $x \in \mathbb{X} \subseteq \mathbb{R}^{n}$ and $u \in \mathbb{U} \subseteq \mathbb{R}^{m}$. We assume that $f$ is continuous in $(x, u)$. The system is subject to (possibly coupled) state and input constraints

$$
(x, u) \in \mathbb{Z}
$$

for some compact set $\mathbb{Z} \subseteq \mathbb{X} \times \mathbb{U}$. Furthermore, the system is subject to average constraints, which are expressed in terms of an auxiliary output variable

$$
y=h(x, u),
$$

where $y \in \mathbb{R}^{p}$ and $h$ is assumed to be continuous in $(x, u)$. Asymptotic average constraints, which were already considered in $[2,7]$, are given as

$$
A v[y] \subseteq \mathbb{Y}
$$

for some closed convex set $\mathbb{Y} \subseteq \mathbb{R}^{p}$. As discussed in the introduction, the novel feature considered in this paper will be transient average constraints, i.e., constraints on input and state variables averaged over some finite time period $T$. Namely, for some given time period $T \geq 1$, we require that the following is satisfied for all $t \geq 0$ :

$$
\sum_{k=t}^{t+T-1} \frac{h(x(k), u(k))}{T} \in \mathbb{Y} .
$$

Note that $T=1$ corresponds to standard pointwise in time constraints, which can be absorbed into (2). Hence in the following, we are interested in the case of $T \geq 2$.

The system (1) is equipped with a continuous stage cost $\ell: \mathbb{Z} \rightarrow \mathbb{R}$, which can be a general, possibly economic, cost function and need not satisfy any specific requirements such as convexity or positive definiteness with respect to any setpoint. Let $\left(x_{s}, u_{s}\right)$ be the optimal steadystate defined as

$$
\ell\left(x_{s}, u_{s}\right)=\min _{(x, u) \in \mathbb{Z}, h(x, u) \in \mathbb{Y}, x=f(x, u)} \ell(x, u) .
$$

Note that the minimum in (5) exists as $\ell$ and $h$ are continuous, $\mathbb{Y}$ is closed and $\mathbb{Z}$ is compact. Furthermore, for simplicity we assume that $\left(x_{s}, u_{s}\right)$ is unique; if this is not the case, in the following let $\left(x_{s}, u_{s}\right)$ denote any of the steady-states satisfying (5).

For the sake of completeness and for a better comparison later on, we now recall the economic MPC algorithm including asymptotic average constraints from $[2$, 7]. Namely, at each time $t$, the following optimization problem is solved:

$$
\min _{\mathbf{u}(t)} \sum_{k=0}^{N-1} \ell(x(k \mid t), u(k \mid t))+V_{f}(x(N \mid t))
$$

subject to

$$
\begin{aligned}
& x(k+1 \mid t)=f(x(k \mid t), u(k \mid t)), \quad k \in \mathbb{I}_{[0, N-1]} \\
&(x(k \mid t), u(k \mid t)) \in \mathbb{Z}, \quad k \in \mathbb{I}_{[0, N-1]} \\
& x(N \mid t) \in \mathbb{X}_{f}, \quad x(0 \mid t)=x(t) \\
& \sum_{k=0}^{N-1} h(x(k \mid t), u(k \mid t)) \in \mathbb{Y}_{t}
\end{aligned}
$$


where $\mathbf{u}(t):=\{u(0 \mid t), \ldots, u(N-1 \mid t)\}$ and $\mathbf{x}(t):=$ $\{x(0 \mid t), \ldots, x(N \mid t)\}$ are the predicted input and corresponding state sequences, $N$ is the prediction horizon, $V_{f}: \mathbb{X} \rightarrow \mathbb{R}$ is the terminal cost function which is assumed to be continuous, and the terminal region $\mathbb{X}_{f} \subseteq \mathbb{X}$ is some compact set containing $x_{s}$. The time-varying output set $\mathbb{Y}_{t}$ is recursively defined as

$$
\begin{aligned}
\mathbb{Y}_{0} & :=N \mathbb{Y} \oplus \mathbb{Y}_{00}, \\
\mathbb{Y}_{t+1} & :=\mathbb{Y}_{t} \oplus \mathbb{Y} \oplus \overline{\mathbb{Y}}(t) \oplus\{-h(x(t), u(t))\},
\end{aligned}
$$

for some $\overline{\mathbb{Y}}(t)$ and some arbitrary compact set $\mathbb{Y}_{00} \subseteq \mathbb{R}^{p}$ containing $h\left(x_{s}, u_{s}\right)$. In [7], it was shown that if the sets $\overline{\mathbb{Y}}(t)$ and $\mathbb{X}_{f}$ (possibly also time-varying) are appropriately defined, then the optimization problem (6)-(7) is recursively feasible and, given any admissible initial condition, for the signals of the resulting closed-loop system it holds that (i) both pointwise in time constraints (2) as well as asymptotic average constraints (3) are satisfied and (ii) the asymptotic average performance is at least as good as the optimal steady-state $\operatorname{cost} \ell\left(x_{s}, u_{s}\right)$, i.e.

$$
A v[\ell(x, u)] \subseteq\left(-\infty, \ell\left(x_{s}, u_{s}\right)\right]
$$

\section{Economic MPC with transient average con- straints}

In this section, we consider economic MPC with transient average constraints of the form (4). As discussed above, such transient average constraints are stricter than asymptotic average constraints of the form (3), and hence also have to be taken into account in a different way within the economic MPC algorithm. In the following, we assume that the set $\mathbb{Y}$ is given as

$$
\mathbb{Y}:=\left\{y \in \mathbb{R}^{p}: y \leq 0\right\}=\mathbb{R}_{\leq 0}^{p} .
$$

Note that this is not a major restriction, as the output map $h$ can be some general nonlinear function. Furthermore, we will impose the following conditions concerning the terminal region $\mathbb{X}_{f}$ and the terminal cost $V_{f}$.

Assumption 1 There exists an auxiliary terminal control law $\kappa_{f}: \mathbb{X} \rightarrow \mathbb{U}$ and a terminal region $\mathbb{X}_{f} \subseteq \mathbb{X}$ containing $x_{s}$ such that for all $x \in \mathbb{X}_{f}$, the following is satisfied: (i) $\left(x, \kappa_{f}(x)\right) \in \mathbb{Z}$, (ii) $f\left(x, \kappa_{f}(x)\right) \in \mathbb{X}_{f}$, and (iii)

$$
V_{f}\left(f\left(x, \kappa_{f}(x)\right)\right)-V_{f}(x) \leq-\ell\left(x, \kappa_{f}(x)\right)+\ell\left(x_{s}, u_{s}\right) .
$$

Assumption 2 The terminal region $\mathbb{X}_{f}$ and the auxiliary terminal control law $\kappa_{f}$ are such that $h\left(x, \kappa_{f}(x)\right) \in$ $\mathbb{Y}$ (i.e., $\left.h\left(x, \kappa_{f}(x)\right) \leq 0\right)$ for all $x \in \mathbb{X}_{f}$.

Remark 1 In [3], under the assumption that $f$ and $\ell$ are twice continuously differentiable and the linearized system around $\left(x_{s}, u_{s}\right)$ is stabilizable, it was shown how $V_{f}$, $\kappa_{f}$ and $\mathcal{X}_{f}$ can be computed in an economic MPC setting such that Assumption 1 is satisfied. Note that this procedure is different than in the (standard) tracking MPC context, as $\ell$ is not assumed to be positive definite with respect to $\left(x_{s}, u_{s}\right)$. Furthermore, Assumption 2 is needed later on in order to ensure recursive feasibility and satisfaction of the transient average constraints (4). Note that Assumption 2 is trivially satisfied if the terminal equality constraint $x(N \mid t)=x_{s}$ is used instead of a terminal region constraint, as $h\left(x_{s}, u_{s}\right) \in \mathbb{Y}$ by definition. In Section 3.1, we present some extensions for the case where this assumption is not satisfied. Finally, as discussed in Section 1, we note that despite the use of a terminal region, the closed-loop system in economic MPC will in general not be convergent due to the use of a general cost function (see, e.g., [2, 3, 7] and Section 4).

The proposed economic MPC algorithm is now given as follows. At each time $t \in \mathbb{I}_{\geq 0}$, solve

$$
\min _{u(0 \mid t), \ldots, u(N-1 \mid t)} \sum_{k=0}^{N-1} \ell(x(k \mid t), u(k \mid t))+V_{f}(x(N \mid t))
$$

subject to

$$
\begin{gathered}
x(k+1 \mid t)=f(x(k \mid t), u(k \mid t)), \quad k \in \mathbb{I}_{[0, N+T-3]} \\
(x(k \mid t), u(k \mid t)) \in \mathbb{Z}, \quad k \in \mathbb{I}_{[0, N-1]} \\
x(N \mid t) \in \mathbb{X}_{f}, \quad x(0 \mid t)=x(t) \\
\sum_{k=t-T+i}^{t-1} h(x(k), u(k))+\sum_{k=0}^{i-1} h(x(k \mid t), u(k \mid t)) \leq 0, \\
i \in \mathbb{I}_{[\max \{1, T-t\}, T]} \\
\sum_{k=j}^{j+T-1} h(x(k \mid t), u(k \mid t)) \leq 0, \quad j \in \mathbb{I}_{[1, N-1]} \\
u(k \mid t)=\kappa_{f}(x(k \mid t)) \quad k \in \mathbb{I}_{[N, N+T-2]}
\end{gathered}
$$

The main novel feature of this algorithm are the constraints (13d)-(13e). Each of these constraints consists of a sum of $T$ addends, and in total ${ }^{1}(N+T-1) p$ such constraints are imposed via (13d)-(13e). In (13d), the terms in the first sum $^{2}$ are the output values along the past closed-loop solution up to time $t-1$, and $\mathbf{u}(t):=\{u(0 \mid t), \ldots, u(N+T-2 \mid t)\}$ and $\mathbf{x}(t):=\{x(0 \mid t), \ldots, x(N+T-2 \mid t)\}$ denote again predicted input and corresponding state sequences, respectively. Note that while predicted inputs and states up to $k=N+T-2$ are now needed in (13e), the number of free input variables is not increased in problem (12)(13), i.e., the minimization is still done over the first $N$ elements $\{u(0 \mid t), \ldots, u(N-1 \mid t)\}$. For $k \geq N$, the predicted inputs and states are fixed by (13f) together with (13a), respectively.

Remark 2 For ensuring asymptotic average constraints, $p$ additional constraints $(7 \mathrm{~d})$ were needed in the optimization problem at each time $t$. On the other hand, in order to ensure satisfaction of transient average constraints, i.e., that (4) holds for all $t \in \mathbb{I}_{\geq 0}$, $(N+T-1) p$ additional constraints (13d)-(13e) are needed. This is due to the fact that as discussed above,

\footnotetext{
1 Recall that $p$ is the dimension of the output map $h$.

${ }^{2}$ By convention, the empty sum is zero (for $i=T$ ).
} 
transient average constraints are stricter than asymptotic average constraints, and hence they also have to be taken into account in a different way within the respective optimization problem. Nevertheless, we again emphasize that the number of free input variables is the same for both problems (6)-(7) and (12)-(13).

Remark 3 Constraint (13e) could be replaced by

$$
\sum_{k=j}^{\min \{j+T-1, N-1\}} h(x(k \mid t), u(k \mid t)) \leq 0, \quad j \in \mathbb{I}_{[1, N-1]},
$$

without changing the properties of the algorithm which are stated below. Then, one would not have to consider predicted input and state variables up to $k=N+T-2$, but only up to $N-1$ (or $N$, respectively) as usual, and hence (13f) could be omitted. However, this alternative formulation is more conservative. Namely, as $x(k \mid t) \in \mathbb{X}_{f}$ and $u(k \mid t)=\kappa_{f}(x(k \mid t))$ for all $k \in \mathbb{I}_{[N, N+T-2]}$ (which is ensured by (13c), (13a) and (13f) together with Assumption 1(ii)), by Assumption 2 it follows that $h(x(k \mid t), u(k \mid t)) \leq 0$ for all $k \in \mathbb{I}_{[N, N+T-2]}$, and hence requiring (13e) to hold is less restrictive than (14).

Remark 4 As already mentioned above, $T=1$ corresponds to usual pointwise in time state and input constraints. Indeed, in this case, (13f) disappears and (13d)(13e) constitute some (additional) pointwise in time constraints (i.e., each constraint of $(13 \mathrm{~d})-(13 \mathrm{e})$ consists of only one term), which can be absorbed into (13b). Furthermore, we note that an alternative way to deal with transient average constraints would be to define the extended state $z(t)=\left[x(t)^{T} \ldots x(t-T+1)^{T}\right]^{T}$ and then use a (standard) economic MPC algorithm for the corresponding extended system including standard pointwisein-time constraints only. However, this alternative formulation has the following drawbacks. First, the terminal constraint for the extended system would in fact require that $x(N-T+1 \mid t), \ldots, x(N \mid t)$ are close to the optimal steady-state $x_{s}$, while via (13c) this is only required for $x(N \mid t)$. Furthermore, the terminal cost $V_{f}$ would then be a function of $x(N-T+1 \mid t), \ldots, x(N \mid t)$ instead of $x(N \mid t)$ only, which means that a different cost than the original (economic) cost $\ell$ is used over this time horizon. Finally, applying stability analysis results for standard economic $M P C$ to the extended system would in general result in a stricter dissipativity requirement than the one used in Theorem 2 (involving the multiplier $\bar{\lambda}$ ). For these reasons, we propose to define the economic MPC algorithm for the original system and take care of the transient average constraints by means of (13d)-(13e).

Let $\mathbf{u}^{0}(t)$ denote the minimizer ${ }^{3}$ of problem (12)-(13) at time $t$, and $\mathbf{x}^{0}(t)$ the corresponding predicted state sequence. As usual in MPC, the input to system (1) at time $t$ is then given by the first element of $\mathbf{u}^{0}(t)$, resulting

\footnotetext{
${ }^{3}$ If $\mathbf{u}^{0}(t)$ is not unique, just assign a unique constant selection map to select one of the multiple minima.
}

in the closed-loop system

$$
x(t+1)=f(x(t), u(0 \mid t)) .
$$

Theorem 1 Suppose that Assumptions 1-2 are satisfied, and that the optimization problem (12)-(13) is feasible at $t=0$. Then (12)-(13) is feasible for all $t \in \mathbb{I}_{\geq 0}$ and the resulting closed-loop system (15) satisfies the pointwise in time constraints (2), the transient average constraints (4) and the asymptotic average performance bound (10).

Proof: The proof of recursive feasibility is as usual by induction. Suppose that the optimization problem (12)-(13) is feasible at time $t$. Then, consider at time $t+1$ the candidate input sequence $\hat{\mathbf{u}}(t+$ 1) $:=\left\{u^{0}(1 \mid t), \ldots, u^{0}(N+T-2 \mid t), \kappa_{f}(\hat{x}(N+T-\right.$ $2 \mid t))\}$ with corresponding state sequence $\hat{\mathbf{x}}(t+1):=$ $\left\{x^{0}(1 \mid t), \ldots, x^{0}(N+T-2 \mid t), f\left(x^{0}(N+T-2 \mid t), \kappa_{f}\left(x^{0}(N+\right.\right.\right.$ $T-2 \mid t)))\}$. For these candidate input and state sequences, constraints (13a)-(13c) and (13f) are satisfied, which follows as in standard MPC feasibility proofs due to Assumption 1 and the fact that they were satisfied at time $t$ (see, e.g., [12]). Now consider constraint (13d) for some $i \in \mathbb{I}_{[\max \{1, T-t\}, T-1]}$. Evaluated for the given candidate input and state sequences, the left-hand side reads

$$
\begin{aligned}
& \sum_{k=t+1-T+i}^{t} h(x(k), u(k))+\sum_{k=0}^{i-1} h(\hat{x}(k \mid t+1), \hat{u}(k \mid t+1)) \\
= & \sum_{k=t+1-T+i}^{t} h(x(k), u(k))+\sum_{k=0}^{i-1} h\left(x^{0}(k+1 \mid t), u^{0}(k+1 \mid t)\right) \\
= & \sum_{k=t-T+(i+1)}^{t-1} h(x(k), u(k))+\sum_{k=0}^{(i+1)-1} h\left(x^{0}(k \mid t), u^{0}(k \mid t)\right)
\end{aligned}
$$

But this means that the $i$-th constraint of (13d) at time $t+1$ corresponds to the $(i+1)$-st constraint of (13d) at time $t$, which was satisfied by assumption. Hence constraint (13d) is satisfied at time $t+1$ for all $i \in \mathbb{I}_{[\max \{1, T-t\}, T-1]}$. Similarly, one can show that constraint (13d) with $i=T$ at time $t+1$ evaluated for the candidate input and state sequences corresponds to constraint (13e) with $j=1$ at time $t$, and that for each $j \in \mathbb{I}_{[1, N-2]}$, constraint (13e) at time $t+1$ evaluated for the candidate input and state sequences corresponds to the $(j+1)$-st constraint of (13e) at time $t$. Finally, when evaluated for the given candidate input and state sequences, constraint (13e) with $j=N-1$ only contains terms $h\left(x, \kappa_{f}(x)\right)$ with $x \in \mathbb{X}_{f}$, which is the case due to (13f) and (13a) and the fact that $\hat{x}(N-1 \mid t+1)=x^{0}(N \mid t) \in \mathbb{X}_{f}$. But then, by Assumption 2 , constraint (13e) with $j=N-1$ is also satisfied at time $t+1$. Hence the candidate input and state sequences $\hat{\mathbf{u}}(t+1)$ and $\hat{\mathbf{x}}(t+1)$, respectively, satisfy the constraints (13), which by induction implies that the optimization problem (12)-(13) is feasible for all $t \in \mathbb{I}_{\geq 0}$.

Satisfaction of the pointwise in time constraints (2) by the closed-loop system (15) immediately follows by defi- 
nition of the receding horizon control law. Fulfillment of the transient average constraints (4) for all $t \in \mathbb{I}_{\geq 0}$ follows directly from the first constraint of (13d) (i.e., with $i=1$ ) and the definition of the receding horizon control law. Namely, from (13d) with $i=1$ (this constraint applies for all $t \geq T-1$ ) and the definition of the receding horizon control law, we obtain that along the closed-loop system (15) the following is satisfied for all $t \geq T-1$ :

$$
\sum_{k=t-T+1}^{t} h(x(k), u(k)) \leq 0 .
$$

By an index shift and considering that $T>0$, this is equivalent to the fact that (4) is satisfied for all $t \in \mathbb{I}_{\geq 0}$. Finally, the asymptotic average performance bound (10) can be established as in $[2,3]$.

Remark 5 Satisfaction of the transient average constraints (4) with some $T \geq 1$ and $\mathbb{Y}$ given by (11) immediately implies that also transient average constraints (4) with period $k T$ are satisfied, for each $k \in \mathbb{I}_{\geq 1}$. Furthermore, also the asymptotic average constraint (3) with $\mathbb{Y}$ given by (11) is satisfied, as for each $t \geq 0$

$$
\begin{aligned}
\sum_{k=0}^{t} \frac{h(x(k), u(k))}{t+1}= & \sum_{i=0}^{\lfloor(t+1) / T\rfloor-1} \sum_{k=i T}^{t(i+1) T-1} \frac{h(x(k), u(k))}{t+1} \\
& +\sum_{k=\lfloor(t+1) / T\rfloor T}^{t} \frac{h(x(k), u(k))}{t+1} \\
& \stackrel{(4)}{\leq} \sum_{k=\lfloor(t+1) / T\rfloor T}^{t} \frac{h(x(k), u(k))}{t+1}
\end{aligned}
$$

Hence, as the sum in (16) contains at most $T-1$ terms and furthermore $h$ is continuous and $\mathbb{Z}$ compact, we obtain that

$$
\begin{aligned}
& \limsup _{t \rightarrow \infty} \sum_{k=0}^{t} \frac{h(x(k), u(k))}{t+1} \\
& \leq \limsup _{t \rightarrow \infty} \sum_{k=\lfloor(t+1) / T\rfloor T}^{t} \frac{h(x(k), u(k))}{t+1}=0,
\end{aligned}
$$

i.e., the asymptotic average constraint (3) is satisfied.

Considering Remark 5, we arrive at the following corollary.

Corollary 1 Suppose that the conditions of Theorem 1 hold. Then for the closed-loop system (15), transient average constraints of the form (4) with period $k T$ are satisfied for each $k \in \mathbb{I}_{\geq 1}$. Furthermore, the asymptotic average constraint (3) with $\mathbb{Y}$ given by (11) is satisfied.

Having established recursive feasibility and satisfaction of the transient average constraints, we now deal with the question when the resulting closed-loop system (15) converges to the optimal steady-state $\left(x_{s}, u_{s}\right)$. As discussed in the introduction, this will in general not be the case as the stage cost $\ell$ is some (arbitrary) cost function which is not necessarily positive definite with respect to $\left(x_{s}, u_{s}\right)$. In [2], it was shown that if a certain dissipativity condition is satisfied, then operation of a system at the optimal steady-state $\left(x_{s}, u_{s}\right)$ is indeed optimal, i.e., no other trajectory results in a better average performance than if the system is at the optimal steady-state $\left(x_{s}, u_{s}\right)$. Furthermore, in $[7,11]$ it was shown that under the same dissipativity condition (strengthened to strict dissipativity), asymptotic convergence of the closed-loop resulting from the economic MPC algorithm (6)-(7), which ensures satisfaction of asymptotic average constraints, follows. We will now show that the same is true for the proposed algorithm (12)-(13) which ensures satisfaction of transient average constraints. To this end, for a set $\mathbb{W} \subseteq \mathbb{Z}$, denote by $\mathbb{W}_{\mathbb{X}}$ the projection of $\mathbb{W}$ on $\mathbb{X}$, i.e., $\mathbb{W}_{\mathbb{X}}:=\{x \in \mathbb{X}: \exists u \in \mathbb{U}$ s.t. $(x, u) \in \mathbb{W}\}$, and consider the following definition of dissipativity $[13,14]$ :

Definition 1 The system (1) is dissipative on a set $\mathbb{W} \subseteq$ $\mathbb{Z}$ with supply rate $s: \mathbb{X} \times \mathbb{U} \rightarrow \mathbb{R}$ if there exists a bounded storage function $\lambda: \mathbb{W}_{\mathbb{X}} \rightarrow \mathbb{R}$ such that the following inequality is satisfied for all $(x, u) \in \mathbb{W}$ :

$$
\lambda(f(x, u))-\lambda(x) \leq s(x, u) .
$$

If, in addition, for some positive definite ${ }^{4} \rho: \mathbb{W}_{\mathbb{X}} \rightarrow \mathbb{R}$ it holds that for all $(x, u) \in \mathbb{W}$

$$
\lambda(f(x, u))-\lambda(x) \leq-\rho(x)+s(x, u),
$$

then system (1) is strictly dissipative on $\mathbb{W}$.

For a further discussion on dissipativity in economic $\mathrm{MPC}$, the interested reader is referred to $[11,15]$. We can now state the following result.

Theorem 2 Suppose that the conditions of Theorem 1 hold, and that there exists a multiplier $\bar{\lambda} \in \mathbb{R}_{\geq 0}^{p}$ such that system (1) is strictly dissipative on $\mathbb{Z}$ with supply rate $s(x, u)=\ell(x, u)-\ell\left(x_{s}, u_{s}\right)+\bar{\lambda}^{T} h(x, u)$. Then the resulting closed-loop system (15) asymptotically converges to $x_{s}$, i.e., $\lim _{t \rightarrow \infty} x(t)=x_{s}$.

Proof: The proof of Theorem 2 follows the lines of the respective proofs in $[7,11]$. There, the function $V(t)=$ $\widetilde{V}_{N}^{0}(x(t))+w(t)$ was used as a Lyapunov-like function, where $\widetilde{V}_{N}^{0}$ is the optimal value function of problem (12)(13), but with $\ell$ and $V_{f}$ in (12) replaced by $\tilde{\ell}$ and $\tilde{V}_{f}$, respectively, where $\tilde{\ell}(x, u):=\ell(x, u)-\ell\left(x_{s}, u_{s}\right)+\lambda(x)-$ $\lambda(f(x, u))$ and $\tilde{V}_{f}:=V_{f}(x)-V_{f}\left(x_{s}\right)+\lambda(x)-\lambda\left(x_{s}\right)$. Furthermore, $w$ is defined as

$$
w(t):=\sup _{\tau \in \mathbb{I} \geq 0} \sum_{k=t}^{t+\tau} \bar{\lambda}^{T} h(x(k), u(k)),
$$

\footnotetext{
4 A function $\rho$ is positive definite with respect to some point $\bar{x} \in \mathbb{X}$ if it is continuous, $\rho(\bar{x})=0$ and $\rho(x)>0$ for all $x \in \mathbb{X}$ with $x \neq \bar{x}$. In the following, when speaking of strict dissipativity, we take $\bar{x}=x_{s}$, i.e., the function $\rho$ is assumed to be positive definite with respect to the optimal steadystate $x_{s}$ defined via (5).
} 
with $h(x(k), u(k))$ being the output along the solution of the resulting closed-loop system (15) from time $t$ on. The only step in the proof which differs from $[7,11]$ is to show that $w(0)<\infty$. In our setting, using similar calculations as in (16) together with the fact that $\bar{\lambda} \in \mathbb{R}_{\geq 0}^{p}$, we obtain that for each $t \in \mathbb{I}_{\geq 0}$ and each $\tau \in \mathbb{I}_{\geq 0}$

$$
\sum_{k=t}^{t+\tau} \bar{\lambda}^{T} h(x(k), u(k)) \leq \sum_{k=t+\lfloor(\tau+1) / T\rfloor T}^{t+\tau} \bar{\lambda}^{T} h(x(k), u(k)) .
$$

Again, the sum on the right hand side consists of at most $T-1$ terms, and hence for each $t \in \mathbb{I}_{\geq 0}$ we have

$$
w(t) \leq(T-1) \max _{(x, u) \in \mathbb{Z}} \bar{\lambda}^{T} h(x, u)<\infty,
$$

where the last inequality follows from continuity of $h$ and compactness of $\mathbb{Z}$. The rest of the proof then follows as in $[7,11]$.

Remark 6 One might suspect that with the help of Corollary 1 (respectively, Remark 5), asymptotic convergence of the closed-loop system could directly be concluded from [7, 11] without any modifications of the proof, as fulfilment of the transient average constraints (4) with some $T \geq 1$ implies satisfaction of the asymptotic average constraints (3). However, this is not possible. Namely, in [7, 11], one crucial step in the respective proofs is to show boundedness of $w(0)$, which is a slightly stronger property than satisfaction of the asymptotic average constraints (i.e., (3) does not necessarily imply boundedness of $w(0))$. In our setting with transient average constraints, boundedness of $w(t)$ for each $t \in \mathbb{I}_{>0}$ can be established as shown above in the proof of Theorem 2. Furthermore, we remark that in case of both transient and asymptotic average constraints, one can in general only establish asymptotic convergence (but not asymptotic stability) of the closed-loop system. This is the case as the average constraints allow the system to initially "spend time" in a region of the state-space where it is not allowed on average (see [7, 11] for a more detailed discussion on this issue). Finally, we note that the function $V$ in the proof of Theorem 2 is different from the Lyapunov function used in economic MPC without average constraints [2, 3]. Namely, in these references, $\widetilde{V}_{N}^{0}(x)$ is used as a Lyapunov function, while here we need the additional term $w$ defined by (19).

\subsection{Relaxed transient average constraints}

In case that Assumption 2 is not satisfied, the above results cannot readily be applied. Namely, the shifted candidate input and state sequences at time $t+1$ used in the proof of Theorem 1 do not necessarily satisfy (13e), as now there exist $x \in \mathbb{X}_{f}$ with $h\left(x, \kappa_{f}(x)\right)>0$. Nevertheless, we will show in the following that the proposed economic MPC algorithm can be modified such that a slightly relaxed form of the transient average constraints (4) can be ensured, namely that for all $t \in \mathbb{I}_{\geq 0}$

$$
\left|\sum_{k=t}^{t+T-1} \frac{h(x(k), u(k))}{T}\right|_{\mathbb{Y}} \leq \sigma(t)
$$

where $\sigma$ is nonincreasing and decaying to zero as $t \rightarrow \infty$. This means that the violation of the original transient average constraints (4) (measured in terms of the distance to the set $\mathbb{Y}$ ) is upper-bounded by the term $\sigma(t)$, which decays to zero as $t \rightarrow \infty$, i.e., asymptotically the original transient average constraints (4) are satisfied. In order to ensure this relaxed form of average constraints, the economic MPC algorithm (12)-(13) is modified as follows. First, constraints $(13 \mathrm{~d})-(13 \mathrm{e})$ are replaced by

$$
\begin{gathered}
\left|\sum_{k=t-T+i}^{t-1} h(x(k), u(k))+\sum_{k=0}^{i-1} h(x(k \mid t), u(k \mid t))\right|_{\mathbb{Y}} \\
\leq \bar{\sigma}(t+i-T-N+1), \quad i \in \mathbb{I}_{[\max \{1, T-t\}, T]} \\
\left|\sum_{k=j}^{j+T-1} h(x(k \mid t), u(k \mid t))\right|_{\mathbb{Y}} \leq \bar{\sigma}(t+j-N+1), \\
j \in \mathbb{I}_{[1, N-1]}
\end{gathered}
$$

where $\bar{\sigma}$ will be defined below. Second, the terminal constraint (13c) will be modified as follows. In order to simplify notation, in the following we assume without loss of generality that $\left(x_{s}, u_{s}\right)=(0,0)$. Furthermore, we make the following assumption concerning the terminal region $\mathbb{X}_{f}$ and the terminal auxiliary control law $\kappa_{f}$.

Assumption 3 The terminal auxiliary control law $\kappa_{f}$ $i s$ continuous. The terminal region $\mathbb{X}_{f}$ is of the form $\mathbb{X}_{f}:=\left\{x \in \mathbb{R}^{n}: V(x) \leq b_{0}\right\}$ for some $b_{0} \geq 0$ and $V(x):=x^{T} P x$ with $P>0$. Furthermore, the following is satisfied: $V\left(f\left(x, \kappa_{f}(x)\right)\right)-V(x) \leq-x^{T} Q x$ for some $Q>0$ and all $x \in \mathbb{X}_{f}$.

Remark 7 The approach proposed in [3] results in a terminal region $\mathbb{X}_{f}$ and a terminal auxiliary control law $\kappa_{f}(x)$ satisfying both Assumptions 1 and 3. In particular, it is also shown how the constant $b_{0}$ can be calculated such that Assumption 1 is satisfied with $\mathbb{X}_{f}:=\left\{x \in \mathbb{R}^{n}: V(x) \leq b\right\}$ for all $0<b \leq b_{0}$.

We now propose to use the following time-varying terminal region $\mathbb{X}_{f}(t)$ instead of the constant terminal region $\mathbb{X}_{f}$ in $(13 \mathrm{c})$ :

$$
\mathbb{X}_{f}(t):=\left\{x \in \mathbb{R}^{n}: V(x) \leq b(t)\right\}, \quad b(0)=b_{0},
$$

where

$$
\begin{aligned}
b(t+1) & =\max \left\{\left(1-\frac{\lambda_{\min }(Q)}{\lambda_{\max }(P)}\right) b(t), \min \left\{b_{0}, b_{\max }\right\}\right\}, \\
b_{\max } & =\sup _{h\left(x, \kappa_{f}(x)\right) \in \mathbb{Y}, \forall x \in\left\{x \in \mathbb{R}^{n}: V(x) \leq b\right\}} b .
\end{aligned}
$$

Such a time-varying terminal region has also been used in the context of economic MPC with asymptotic average constraints (see [7, Section 3.2]). Note that $b_{\max } \geq 0$ as the optimal steady-state satisfies $h\left(x_{s}, u_{s}\right) \in \mathbb{Y}$ by definition (see (5)). If $b_{0} \leq b_{\max }$, then $\mathbb{X}_{f}(t)=\mathbb{X}_{f}(0)$ for all $t \in \mathbb{I}_{\geq 0}$ and Assumption 2 is satisfied, i.e., Theorem 1 can be applied. Otherwise, the definition of $\mathbb{X}_{f}(t)$ in (23)-(24) means that the time-varying terminal region is gradually tightened such that ultimately (at least 
asymptotically) Assumption 2 is satisfied. In case that $b_{\max }>0$, there exists some finite time $t^{\prime} \in \mathbb{I}_{\geq 0}$ such that the terminal region is constant for all $t \in \mathbb{I}_{\geq t^{\prime}}$ and given by $\mathbb{X}_{f}(t)=\left\{x \in \mathbb{R}^{n}: V(x) \leq b_{\max }\right\}$. In fact, in this case one could also use the (constant) terminal region $\mathbb{X}_{f}(t)=\left\{x \in \mathbb{R}^{n}: V(x) \leq b_{\max }\right\}$ for all $t \in \mathbb{I}_{\geq 0}$. However, using the time-varying terminal regions (23)-(24) results in a possibly larger feasible region (in case that $\left.b_{0}>b_{\max }\right)$, as the set of all fesible initial conditions $x_{0}$ when using the time-varying terminal regions (23)-(24) is equal to that of an MPC algorithm using the fixed terminal region $\mathbb{X}_{f}=\left\{x \in \mathbb{R}^{n}: V(x) \leq b_{0}\right\}$ (compare Theorem 3).

Finally, we note that due to continuity of $h$ and $\kappa_{f}$, also $\left|h\left(x, \kappa_{f}(x)\right)\right|_{\mathbb{Y}}$ is continuous in $x$; hence, due to the fact that $h\left(x_{s}, u_{s}\right) \in \mathbb{Y}$, there exists a function $\gamma \in \mathcal{K}$ such that

$$
\left|h\left(x, \kappa_{f}(x)\right)\right|_{\mathbb{Y}} \leq \gamma(V(x))
$$

for all $x \in \mathbb{X}_{f}(0)$. For example, one could take $\gamma(r):=$ $\max _{V(x) \leq r}\left|h\left(x, \kappa_{f}(x)\right)\right|_{\mathbb{Y}}+r$. We can now state the following result.

Theorem 3 Suppose that Assumptions 1 and 3 are satisfied, and consider the optimization problem (12)-(13) with $\mathbb{X}_{f}$ in (13c) replaced by the time-varying terminal region $\mathbb{X}_{f}(t)$ defined via $(23)-(24)$, and $(13 \mathrm{~d})-(13 \mathrm{e})$ replaced by $(21)-(22)$ with arbitrary $\bar{\sigma}(-N+1), \ldots, \bar{\sigma}(0) \in$ $\mathbb{R}_{\geq 0}$ and

$$
\bar{\sigma}(t):=\sum_{k=t-1}^{t-2+T} \gamma\left(b_{0}\left(1-\frac{\lambda_{\min }(Q)}{\lambda_{\max }(P)}\right)^{k}\right), t \in \mathbb{I}_{\geq 1}
$$

where $\gamma \in \mathcal{K}$ satisfies (25). If this modified optimization problem is feasible at time $t=0$, then it is feasible for all $t \in \mathbb{I}_{\geq 0}$ and the resulting closed-loop system (15) satisfies the pointwise in time constraints (2), the asymptotic average performance bound (10) and the (relaxed) transient average constraints (20) with $\sigma$ given by

$$
\sigma(t)=(1 / T) \bar{\sigma}(t-N+1)
$$

Remark 8 In order obtain the tightest results via (27), one might want to choose small initial values $\bar{\sigma}(-N+$ $1), \ldots, \bar{\sigma}(0)$. On the other hand, they have to be large enough such that the constraints (21)-(22) are initially feasible. Furthermore, $\sigma$ given by $(27)$ is nonincreasing if one chooses $\bar{\sigma}(-N+1) \geq \cdots \geq \bar{\sigma}(0) \geq \bar{\sigma}(1)$, and we have $\lim _{t \rightarrow \infty} \sigma(t)=0$ as required.

Proof of Theorem 3: For the proof of recursive feasibility, at time $t+1$ consider again the candidate input and state sequences $\hat{\mathbf{u}}(t+1)$ and $\hat{\mathbf{x}}(t+1)$ as specified in the proof of Theorem 1. We have to show that both the modified terminal constraint as well as (21)-(22) are feasible, given that the modified optimization problem was feasible at time $t$. By Assumption 3, we have that

$$
\begin{aligned}
& V(\hat{x}(N \mid t+1))=V\left(f\left(x^{0}(N \mid t), \kappa_{f}\left(x^{0}(N \mid t)\right)\right)\right. \\
& \leq V\left(x^{0}(N \mid t)\right)-x^{0}(N \mid t)^{T} Q x^{0}(N \mid t) \\
& \leq\left(1-\frac{\lambda_{\min }(Q)}{\lambda_{\max }(P)}\right) V\left(x^{0}(N \mid t)\right) \leq\left(1-\frac{\lambda_{\min }(Q)}{\lambda_{\max }(P)}\right) b(t),
\end{aligned}
$$

where the last inequality follows from the definition of $\mathbb{X}_{f}(t)$ and the fact that $x^{0}(N \mid t) \in \mathbb{X}_{f}(t)$. Hence $\hat{x}(N \mid t+1) \in \mathbb{X}_{f}(t+1)$ as desired due to the definition of $\mathbb{X}_{f}(t+1)$ according to (23)-(24). Feasibility of the constraints (21) for all $i$ and (22) for $j \in \mathbb{I}_{[1, N-2]}$ can be established in the same way as in the proof of Theorem 1. Finally, for constraint (22) with $j=N-1$, consider the following. By the same reasoning as in (28), we conclude that for each $t \in \mathbb{I}_{\geq 0}$ and each $x \in \mathbb{X}_{f}(t)$, we have that $f\left(x, \kappa_{f}(x)\right) \in \mathbb{X}_{f}(t+1)$. Thus, due to the fact that $\hat{x}(N-1 \mid t+1)=x^{0}(N \mid t) \in \mathbb{X}_{f}(t)$, by $(13 \mathrm{a})$ and (13f) we conclude that $\hat{x}(N+k \mid t+1) \in \mathbb{X}_{f}(t+k+1)$ for all $k \in \mathbb{I}_{[-1, T-2]}$. Now consider the following claim.

Claim 1: For each $t \in \mathbb{I}_{\geq 0}$, the terminal region $\mathbb{X}_{f}(t)$ is such that either $b(t)=b_{0}\left(1-\frac{\lambda_{\min }(Q)}{\lambda_{\max }(P)}\right)^{t}$ or $h\left(x, \kappa_{f}(x)\right) \in$ $\mathbb{Y}$ for all $x \in \mathbb{X}_{f}(t)$.

The proof of this claim follows immediately from the definitions of $\mathbb{X}_{f}(t), b(t)$ and $b_{\max }$ in (23)-(24). Considering the above, we obtain

$$
\begin{aligned}
& \left|\sum_{k=N-1}^{N+T-2} h(\hat{x}(k \mid t+1), \hat{u}(k \mid t+1))\right|_{\mathbb{Y}} \\
& \leq \sum_{k=N-1}^{N+T-2}|h(\hat{x}(k \mid t+1), \hat{u}(k \mid t+1))|_{\mathbb{Y}} \\
& \leq \sum_{k=N-1}^{N+T-2} \gamma\left(b_{0}\left(1-\frac{\lambda_{\min }(Q)}{\lambda_{\max }(P)}\right)^{t+k-N+1}\right)=\bar{\sigma}(t+1),
\end{aligned}
$$

where the first inequality follows due to the definition of $\mathbb{Y}$ in (11), the second inequality follows from Claim 1 and (25) (by considering that $\mathbb{X}_{f}(t) \subseteq \mathbb{X}_{f}(0)$ for all $t \in$ $\mathbb{I}_{\geq 0}$ and $\hat{x}(N+k \mid t+1) \in \mathbb{X}_{f}(t+k+1)$ for all $k \in \mathbb{I}_{[-1, T-2]}$ as established above), and the last equality follows by definition of $\bar{\sigma}$ in (26). This means that constraint (22) with $j=N-1$ is also satisfied at time $t+1$, and hence recursive feasibility is established.

Fulfillment of the pointwise in time constraints (2) and the asymptotic average performance bound (10) for the resulting closed-loop system (15) follow as in Theorem 1. Finally, fulfillment of the (relaxed) transient average constraints (20) for all $t \in \mathbb{I}_{\geq 0}$ follows directly from the first constraint of (21) (i.e., with $i=1$ ) and the definition of the receding horizon control law. Namely, from (21) with $i=1$ (this constraint applies for all $t \geq T-1$ ) and the definition of the receding horizon control law, we obtain that along the closed-loop solution the following is 
satisfied for all $t \geq T-1$ :

$$
\left|\sum_{k=t-T+1}^{t} h(x(k), u(k))\right|_{\mathbb{Y}} \leq \bar{\sigma}(t-T-N+2) .
$$

Dividing by $T>0$ and applying the index shift $t^{\prime}=$ $t-T+1$, this is equivalent to the fact that (20) is satisfied for all $t \in \mathbb{I}_{\geq 0}$ with $\sigma(t)$ given by (27).

Remark 9 As discussed above, in case that $b_{\max }>0$, there exists some finite time $t^{\prime} \in \mathbb{I}_{\geq 0}$ such that $\mathbb{X}_{f}(t)=$ $\overline{\mathbb{X}}_{f}:=\left\{x \in \mathbb{R}^{n}: V(x) \leq \min \left\{b_{0}, b_{\max }\right\}\right\}$ for all $t \in \mathbb{I}_{\geq t^{\prime}}$, which by definition of $b_{\max }$ implies that $h\left(x, \kappa_{f}(x)\right) \in \mathbb{Y}$ for all $x \in \overline{\mathbb{X}}_{f}$. Hence for all $t \in \mathbb{I}_{\geq t^{\prime}+1}$, one can define $\bar{\sigma}(t)=0$ instead of $(26)$ and the results of Theorem 3 still hold. Using (27), this means that the original transient average constraints (4) are satisfied for all $t \in \mathbb{I}_{\geq t^{\prime}+N}$.

\section{Application to a CSTR}

Consider a nonlinear continuous flow stirred-tank reactor with parallel reactions $R \rightarrow P_{1}$ and $R \rightarrow P_{2}$, where $P_{1}$ is the desired product and $P_{2}$ is the waste product [16], which has also been considered previously in the context of asymptotic average constraints [7]. We assume that the first reaction $R \rightarrow P_{1}$ is of second order, whereas the second one $R \rightarrow P_{2}$ is of first order. The dimensionless heat and mass balances for this problem are (see [16] for details)

$$
\begin{aligned}
& \dot{x}_{1}=1-10^{4} x_{1}^{2} e^{-1 / x_{3}}-400 x_{1} e^{-0.55 / x_{3}}-x_{1} \\
& \dot{x}_{2}=10^{4} x_{1}^{2} e^{-1 / x_{3}}-x_{2} \\
& \dot{x}_{3}=u-x_{3}
\end{aligned}
$$

where $x_{1}$ is the concentration of the component $R, x_{2}$ is the concentration of the desired product $P_{1}$ and $x_{3}$ is the temperature of the mixture in the reactor. The manipulated variable $u$ is defined as $u=c_{1}+c_{2} Q$ for some $c_{1}, c_{2}>0$, where $Q$ is the heat flux through the reactor wall (see [16, Appendix I]); given the above used parameter values, $u$ is constrained to lie between 0.049 and 0.449 , while $x$ is considered non-negative. The control objective is to maximize the amount of product $P_{1}$, which translates into a stage cost function $\ell(x, u)=$ $-x_{2}$. The steady-state problem (5) has a solution $x_{s}=$ $\left[\begin{array}{lll}0.0832 & 0.0846 & 0.1491\end{array}\right]^{T}$ and $u_{s}=0.1491$; as was shown in [16], periodic solutions can outperform steady-state operation of the system.

The system is discretized with a sample time $T_{s}=1 / 10$, and a prediction horizon of $N=50$ is chosen; the simulations were implemented in Matlab using integrators from the ACADO toolkit [17]. The terminal cost, region and auxiliary control law were calculated such that Assumptions 1 and 2 are satisfied. Figure 1(a) shows simulation results for the input $u$ and the concentration of the desired product $x_{2}$, if no average constraints are applied. Now assume that transient average constraints of the form (4) are imposed, with $h(x, u)=\left(u-u_{s}\right)^{2}-0.01$
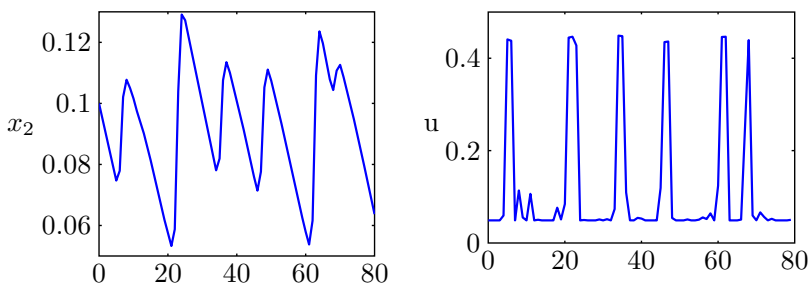

(a) Without average constraints.
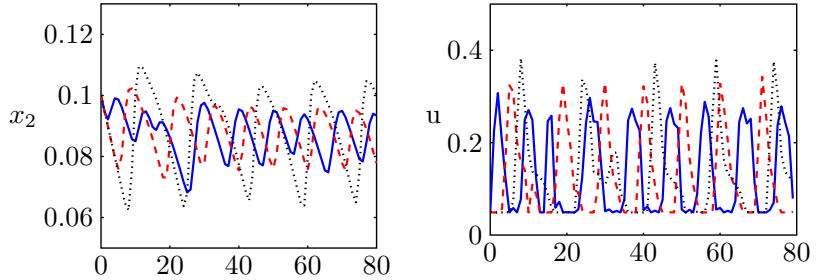

(b) With average constraints and $T=5$ (solid), $T=10$ (dashed) and $T=15$ (dotted).

Fig. 1. Closed-loop input and state $\left(x_{2}\right)$ sequences with different values of $T$.

and $\mathbb{Y}$ given by (11). This means that the deviation of the heat flux $Q$ compared to its value at the optimal steady-state, averaged over $T$ time instants, has to be bounded by some constant. Figure 1(b) shows simulation results for different $T$. For smaller values of $T$, the imposed transient average constraints are stricter (as $h$ averaged over a smaller period has to be nonpositive) and hence result in a more restrictive solution, i.e., the resulting closed-loop sequences for $x_{2}$ and $u$ are shifted towards smaller $x_{2}$ values and less deviation from $u_{s}$, respectively. This can also be seen when evaluating the closed-loop performance, i.e., computing $J=\sum_{t=0}^{t_{\text {end }}}-x_{2}(t)$, where $t_{\text {end }}$ is the simulation end time. We obtain $J=-7.385$ without average constraints, $J=-7.1515$ for $T=15, J=-7.1207$ for $T=10$ and $J=-7.0911$ for $T=5$.

\section{Conclusions}

In this paper, we proposed an economic model predictive control algorithm which ensures fulfillment of transient average constraints. We showed how the repeatedly solved optimization problem has to be defined such that the resulting closed-loop system satisfies such constraints. Furthermore, it turns out that certain properties such as closed-loop average performance bounds and convergence to the optimal steady-state (under a dissipativity condition), which had previously been established for economic MPC with asymptotic average constraints, can be transferred to the proposed setting with transient average constraints. In conclusion, we find that the concept of economic MPC with transient average constraints is relevant in various application contexts, which we exemplarily illustrated with a chemical reactor example. 


\section{References}

[1] M. A. Müller, D. Angeli, and F. Allgöwer, "Economic model predictive control with transient average constraints," in Proceedings of the 52nd IEEE Conference on Decision and Control, 2013, pp. 5119-5124.

[2] D. Angeli, R. Amrit, and J. B. Rawlings, "On average performance and stability of economic model predictive control," IEEE Transactions on Automatic Control, vol. 57, no. 7, pp. 1615-1626, 2012.

[3] R. Amrit, J. B. Rawlings, and D. Angeli, "Economic optimization using model predictive control with a terminal cost," Annual Reviews in Control, vol. 35, no. 2, pp. 178-186, 2011.

[4] L. Grüne, "Economic receding horizon control without terminal constraints," Automatica, vol. 49, no. 3, pp. 725734, 2013.

[5] R. Huang, E. Harinath, and L. T. Biegler, "Lyapunov stability of economically oriented NMPC for cyclic processes," Journal of Process Control, vol. 21, no. 4, pp. 501-509, 2011.

[6] M. Heidarinejad, J. Liu, and P. D. Christofides, "Economic model predictive control of nonlinear process systems using Lyapunov techniques," AIChE Journal, vol. 58, no. 3, pp. 855-870, 2012

[7] M. A. Müller, D. Angeli, F. Allgöwer, R. Amrit, and J. B. Rawlings, "Convergence in economic model predictive control with average constraints," Automatica, 2014, provisionally accepted for publication.

[8] T. G. Hovgaard, L. F. S. Larsen, and J. B. Jørgensen, "Flexible and cost efficient power consumption using economic MPC - a supermarket refrigeration benchmark," in Proceedings of the 50th IEEE Conference on Decision and Control and European Control Conference, 2011, pp. 848854.

[9] W.-J. Ma and V. Gupta, "Desynchronization of thermallycoupled first-order systems using economic model predictive control," in Proceedings of the 51st IEEE Conference on Decision and Control, 2012, pp. 278-283.

[10] B. Chu, S. Duncan, A. Papachristodoulou, and C. Hepburn, "Using economic model predictive control to design sustainable policies for mitigating climate change," in Proceedings of the 51st IEEE Conference on Decision and Control, 2012, pp. 406-411.

[11] M. A. Müller, D. Angeli, and F. Allgöwer, "On convergence of averagely constrained economic MPC and necessity of dissipativity for optimal steady-state operation," in Proceedings of the American Control Conference, 2013, pp. 3147-3152.

[12] D. Q. Mayne, J. B. Rawlings, C. V. Rao, and P. O. M. Scokaert, "Constrained model predictive control: Stability and optimality," Automatica, vol. 36, no. 6, pp. 789-814, 2000 .

[13] J. C. Willems, "Dissipative dynamical systems - part i: General theory," Archive for Rational Mechanics and Analysis, vol. 45, no. 5, pp. 321-351, 1972.

[14] C. I. Byrnes and W. Lin, "Losslessness, feedback equivalence, and the global stabilization of discrete-time nonlinear systems," IEEE Transactions on Automatic Control, vol. 39, no. 1, pp. 83-98, 1994.

[15] M. A. Müller and F. Allgöwer, "Robustness of steadystate optimality in economic model predictive control," in Proceedings of the 51st IEEE Conference on Decision and Control, 2012, pp. 1011-1016.

[16] J. E. Bailey, F. J. M. Horn, and R. C. Lin, "Cyclic operation of reaction systems: Effects of heat and mass transfer resistance," AIChE Journal, vol. 17, no. 4, pp. 818-825, 1971.
[17] B. Houska, H. J. Ferreau, and M. Diehl, "Acado toolkit - an open-source framework for automatic control and dynamic optimization," Optimal Control Applications and Methods, vol. 32 , no. 3, pp. 298-312, 2011. 